This document was prepared in conjunction with work accomplished under Contract No. DE-AC09-96SR18500 with the U.S. Department of Energy.

This work was prepared under an agreement with and funded by the U.S. Government. Neither the U. S. Government or its employees, nor any of its contractors, subcontractors or their employees, makes any express or implied: 1 . warranty or assumes any legal liability for the accuracy, completeness, or for the use or results of such use of any information, product, or process disclosed; or 2 . representation that such use or results of such use would not infringe privately owned rights; or 3 . endorsement or recommendation of any specifically identified commercial product, process, or service. Any views and opinions of authors expressed in this work do not necessarily state or reflect those of the United States Government, or its contractors, or subcontractors. 
WSRC-STI-2008-0351

\title{
Characterization of Detector Grade CdZnTe Material from Redlen Technologies
}

\author{
Martine C. Duff*, Savannah River Nat. Laboratory, Aiken, SC 29808 \\ Arnold Burger, Michael Groza, and Vladimir Buliga, Fisk University, Nashville, TN 37208-3051 \\ John P. Bradley, Zurong R. Dai and Nick Teslich, Lawrence Livermore Nat. Laboratory, \\ Livermore, CA 94550
}

David R. Black, National Institute of Standards and Technology, Gaithersburg, MD 20899 Salah A. Awadalla, Jason Mackenzie and Henry Chen, Redlen Technologies, Sidney, B.C., Canada V8L 5 Y8

\begin{abstract}
CdZnTe (or CZT) crystals can be used in a variety of detector-type applications. This large band gap material shows great promise for use as a gamma radiation spectrometer. Historically, the performance of CZT has typically been adversely affected by point defects, structural and compositional heterogeneities within the crystals, such as twinning, pipes, grain boundaries (polycrystallinity) and secondary phases (SP). The synthesis of CZT material has improved greatly with the primary performance limitation being attributed to mainly SP. In this presentation, we describe the extensive characterization of detector grade material that has been treated with post growth annealing to remove the SPs. Some of the analytical methods used in this study included polarized, cross polarized and transmission IR imaging, I-V curves measurements, synchrotron X-ray topography and electron microscopy.
\end{abstract}

KEYWORDS: Traveling heater method, topography, TEM, hydrogen peroxide etch, secondary phases 


\section{WSRC-STI-2008-0351}

\section{INTRODUCTION}

Synthetic $\mathrm{Cd}_{1-\mathrm{x}} \mathrm{Zn}_{\mathrm{x}} \mathrm{Te}$ or "CZT" crystals can be used for the room temperature-based detection of $\gamma$-radiation. However, the radiation detection properties of CZT crystals vary widely. These variances are not completely understood. They have been attributed to a combination of factors such as point defects, structural and morphological heterogeneities within the crystals, such as twinning, pipes, grain boundaries (polycrystallinity) and secondary phases (SP). ${ }^{1-5}$ During the past 15 years of CZT development, considerable improvements have been made. As the size of useful detectors gradually increased from a few $\mathrm{mm}^{3}$ to a few $\mathrm{cm}^{3}$, the limiting factor in the detector performance seemed to vary from electron and hole trapping by point defects, pipes and cracks to what has more recently been named in the literature as either Te inclusions [as recently described by Carini et al. (2006), Bolotnikov et al. (2007) and refs. therein or as Te precipitates. ${ }^{6-8}$ An explanation for formation of these Te-rich phases is due to the presence of excess Te in the melt of CdTe and CZT phases. Even if the starting charge is stoichiometric or slightly Cd-rich, elemental $\mathrm{Cd}$, which is the most volatile species will preferentially evaporate into the free space of the ampoule and leave behind a Te-rich melt. Tellurium-rich phases are thought to form during crystal growth or crystal annealing due to the retrograde solubility within the phase diagram. ${ }^{9-10}$ Such phases were noted by Rai and researchers (1991) when they observed the presence of many $<0.1 \mu \mathrm{m}$ sized Te-rich SP (called precipitates) in these CdTe materials. ${ }^{11}$

One of the early improvements in the CZT technology addressed the issue of the cracks and the voids within pipes. In recent years these performance limiting features have almost been eliminated from the boules grown with current methods. Fortunately, crystals made using low pressure vertical Bridgman (MVB, by Yinnel Tech in the USA) $)^{12}$ and traveling heater method [THM] (by Redlen in Canada) ${ }^{13,14}$ growth processes provide good control of the growth and these methods produce large single crystals by seeding or self seeding.

\section{EXPERIMENTAL}

The three CZT crystals with similar dimensions (MC3466, MC2539 and 60439) that we characterized in this study were grown at Redlen Technologies (Sidney, B.C., Canada) using the Traveling Heater Method (THM). ${ }^{13-16}$ In this method, Cadmium Zinc Telluride $\left(\mathrm{Cd}_{0.4} \mathrm{Zn}_{0.1} \mathrm{Te}_{0.5}-\mathrm{CZT}\right)$ single crystal boules up to $85 \mathrm{~mm}$ in diameter and 100 $\mathrm{mm}$ long are produced. Crystals are grown epitaxially onto single crystal seeds in the preferred $<111>$ growth direction from a Tellurium solution. The solution and feed compositions are controlled to provide a homogenous axial and radial 


\section{WSRC-STI-2008-0351}

Zinc concentration and metals used are generally $>5 \mathrm{~N}$ purity. Homogenous $\mathrm{Zn}$ concentration is essential to provide uniform electrical properties. The solution and feed are also doped with Indium to compensate for electrical defects related to $\mathrm{Cd}$ loss during growth and annealing. Zinc concentration $\sim 10 \%\left(\mathrm{Cd}_{1-\mathrm{x}} \mathrm{Zn} \mathrm{x}\right.$, with $\left.\mathrm{x}=0.1\right)$ has been measured using Energy Dispersive X-ray analysis (EDX) at the University of British Columbia and Room Temperature Photoluminescence (RTPL) at the Canadian National Research Council (CNRC). ${ }^{13,14}$ Grown boules are wafered to appropriate thicknesses to provide a final detector thickness ranging from 5 to $15 \mathrm{~mm}$. Wafers are subsequently annealed at different temperatures and atmospheres, over selected durations to provide application specific CZT for detector fabrication. Initial solvent and feed composition and doping combined with downstream annealing and fabrication processes provide high resistivity CZT useful for ionizing radiation detection including gamma and X-ray spectroscopy and mapping. The samples used in this study were produced from such THM CZT process just mentioned.

The current-voltage (I-V) curves were obtained using the Keithley 237 Power Unit assisted by a PC computer using the ICS (Interactive Characterization Software) software of Metrics Technology Inc. After polishing, these crystals were characterized by unpolarized and cross polarized IR imaging using a CCD camera for image recording. They were then prepared with gold $\mathrm{Au}$ ) contacts deposited by sputtering in vacuum. The Au contacts allowed for detector performance testing in a planar geometry using a ${ }^{137} \mathrm{Cs}$ gamma source and I-V curve measurements.

X-ray topography (XRT) imaging studies were performed at the Advanced Photon Source at Argonne National Laboratory (Argonne, IL) using beam line 33-BM. An incident energy of $9 \mathrm{keV}$ was selected from the white radiation source using a double crystal monochromator with Si (111) crystals. A rotating foam disc located between the sample and monochromator functioned as a random phase object to remove structure in the incident beam due to phase contrast resulting from imperfections in the beam line Be windows. For these measurements, the sample faces were parallel to (111) and the symmetric (333) images were recorded from these faces. The film was positioned parallel to the sample surface so that the recorded images would not have any foreshortening. Images were recorded on a high contrast lithographic film and in all images the diffraction vector points out of the image.

After the above characterization, half of sample Mc2539 was exposed to concentrated (31.9\% hydrogen peroxide $\left(\mathrm{H}_{2} \mathrm{O}_{2}\right.$, an etchant) for 5 minutes and the other half was left untreated. This material was sent to LLNL for examination by SEM using an FEI Nova 600 Nanolab Dualbeam Focussed Ion Beam Scanning Electron Microscope (FIB-SEM) at $5 \mathrm{keV}$ to analyze and produce thin sections of SP-based areas of interest (through ion-beam milling) for 


\section{WSRC-STI-2008-0351}

high resolution-transmission electron microscopy (HR-TEM) studies. HR-TEM studies were performed with a $200 \mathrm{keV}$ FEI Technai20 G2 FEG monochromated scanning transmission electron microscope (STEM) with high angle annular dark field (HAADF) detector with a $\mathrm{Si}(\mathrm{Li})$ solid state X-ray detector (0.3 steradians solid angle).

\section{RESULTS AND DISCUSSION}

The I-V curve measurements for the three materials reveal that the materials have high resistivities (Fig. 1ac)_ranging from $\rho v=2.0$ to $7.3 \times 10^{10} \Omega \mathrm{cm}$. We determined the performance of these three crystals as gamma radiation spectrometers with the use of a ${ }^{137} \mathrm{Cs}$ source and calculated the full width half maximum (FWHM) of the 662 $\mathrm{keV}$ energy for this decay. All three materials performed well as radiation spectrometers in these studies, with FWHM values between 1.6 and $1.9 \%$ and peak to Compton ratios between 1.9 and 2.9.

Unpolarized IR light was used to examine for cracks, grain boundaries and others major defects in their volume as shown in Fig. 3a-c. MC3466 possesses a possible twin boundary or crack which is more evident than that found with sample 60439. Sample MC2539 is relatively defect-free in comparison to the other two samples. Cross polarized IR light topography was used to detect internal stress due to the growth and the post growth treatments of CZT crystals (based on birefringence of CZT when a mechanical or electrical stress is applied). These images are shown in Fig. 4a-c. IR (unpolarized) microscopic imaging was used to detect the presence of SP, their size and their distribution-see Fig. 5a-c. For all samples there were only a few clusters of SP that were detectable by IR (i.e., those with a size $>1 \mu \mathrm{m})$ so the information about their size and density distribution were not estimated.

X-ray topographic images of the three Redlen CZT materials are shown in Fig. 6a-d. Extremely large angular variation (inferior quality) exists in the MC3466 material (Fig. 6a) relative to that of the MC2539 material (shown in Fig. 6d). The degree of strain is best demonstrated by not only the number of exposures required to record the diffraction-based image of the $1 \mathrm{~cm}^{3}$ crystal but the degrees of rotation required to make the multi-exposure image resolved on the film. The amount of strain present in MC3466 (5 exposures with $0.20^{\circ}$ angles of rotation between each exposure) is considerably greater than that of MC2539 (6 exposures with $0.03^{\circ}$ angles of rotation between each exposure). In contrast, sample 60439 (fig. 6c required one exposure to create a suitable image, which indicates that this material is not as strained as the other two Redlen materials we examined. 


\section{WSRC-STI-2008-0351}

As previously mentioned, the examination of MC3466 with cross polarized IR light (Fig. 4a) reveals striations that were not present in the other two Redlen CZT materials (Fig. 4b-c) that were examined. These striations in MC3466 may be associated with the high degree of crystal strain that is detected in the topography studies with MC3466. Although these images indicate that the MC3466 material is highly strained relative to MC2539, this high degree of strain does not have an adverse effect on radiation detector performance as shown in Fig. 2b. All three materials exhibit high $\gamma$-radiation performance with high resistivities despite differences in crystal strain and crossed polarize IR imaging. Annealing of MVB grown CZT in a Cd- and Zn-rich vapor was found by Zeng and researchers ${ }^{17}$ to result in a reduction in crystal stress and strain. However in our study, we did not examine our materials prior to annealing but our annealing process did not always produce unstrained material or material with poor performance despite the presence of crystalline strain.

Large heterogeneities (100 to $400 \mu \mathrm{m}$ ) of what resemble SP are detected in materials 60439 and MC2539 (in Fig. $6 \mathrm{c}$ and d). The penetration depth of this XRT technique that is only a few tens of $\mu \mathrm{m}$ into the surface and with transmission IR imaging, these SP features should have been readily apparent. For 64039, it is possible that the SP-like features (shown in the topograph in Fig. 6c) may be the same features that are slightly visible in the IR transmission image as shown in Fig. 5c. However, in general, these particularly large features were not detected with transmission IR imaging (Fig. 5a-c). Because the post growth annealing process is performed to remove the SP features in the Redlen CZT material, we consider the features in the XRT studies to be "vestiges" of SP. These vestiges are only apparent in the topography because the annealing process does not remove the crystalline strain (deformation) that was associated with the SP prior to their removal by annealing.

Our more detailed examination with the MC2539 material with SEM (see Fig. 7) did not reveal the presence of faceted SP, such as those found with detector grade CZT made by modified vertical Bridgman (MVB) growth methods at Yinnel Tech. ${ }^{18}$ Additionally, the SP or evidence of their vestiges were not apparent on the CZT surface based on SEM imaging - in contrast to the XRT studies. In general, our SEM studies reveal that the untreated surface material was considerably homogeneous. To examine the near surface environment of this CZT, we selected a region for thin section preparation using surface material that was polished with fine alumina with no etching treatment; the other half of the thin sectioned material was polished and later etched with aqueous $\mathrm{H}_{2} \mathrm{O}_{2}$.

Surface passivation of CZT can be accomplished by etching with aqueous solutions of $\mathrm{H}_{2} \mathrm{O}_{2}{ }^{19}$ or by bombardment with atomic oxygen ${ }^{20}$. When performed after a bromine:methanol etch, the etching of CZT with 


\section{WSRC-STI-2008-0351}

concentrated $\mathrm{H}_{2} \mathrm{O}_{2}$ solutions has been shown to decrease surface leakage currents. ${ }^{21}$ Treatment of CZT with $\mathrm{H}_{2} \mathrm{O}_{2}$ is thought to result in the formation of a high resistivity coating of $\mathrm{CdTeO}$, according to phase diagram predictions. ${ }^{22}$ Our study indicates that the peroxide treatment did not significantly change the Cd:Te composition of the CdZnTe surface and this observation is consistent with but not conclusive that a $\mathrm{CdTeO}$ phase has formed on the CZT surface. The STEM-EDS analyses (see Fig. 8a-e) with MC2539 show no detectable difference of composition between $\mathrm{H}_{2} \mathrm{O}_{2}$-etched region of top surface (Area 4) and un-etched region (Area 3). However, the atomic ratio of $\mathrm{Cd} / \mathrm{Te}$ on top surface (Areas 3 and 4) is slightly lower ( $5 \%$ lower) than that of the CZT bulk (Area 2). SEM imaging studies (Fig. 7) with MC2539 reveal visual differences between the $\mathrm{H}_{2} \mathrm{O}_{2}$-treated surface and the untreated surface-indicating that the surface properties were altered by contact by $\mathrm{H}_{2} \mathrm{O}_{2}$. A closer examination of a thin section made of the treated surface was made using focused ion beam to remove a electron-thin sample for TEM analyses that contained regions with both treatments (etch and no etch)-in addition to some intact subsurface material (a few $\mu \mathrm{m}$ deep) as well as previously mentioned.

TEM bright-field imaging of the top surface of MC2539 is shown in Fig. 9. It appears the surface on the side etched by $\mathrm{H}_{2} \mathrm{O}_{2}$ is a little bit smoother than that on un-etched side. There is a strain layer with a thickness of 0.25 to 1.5 $\mu \mathrm{m}$ below the top surface - regardless of treatment. This strain may be may be introduced during cutting and/or polishing. In general, we could not detect much of an effect (chemical, structural or morphological) of the peroxide etch treatment. This suggests that the oxidizing effect of the treatment remains close to the surface, which is consistent with observations by Bartlett and researchers (1996) who observed a surface oxidation layer thickness of a few tens of nm. ${ }^{18}$

A homogeneous distribution of nanosized SP containing metallic Te have been observed with high resolution TEM in MVB grown CZT that was not annealed. ${ }^{23}$ However, our TEM studies did not detect SP of that nature in the annealed samples. We anticipate that if these features were present prior to the annealing process, they would have been removed upon anneal, much like the large $(>100 \mu \mathrm{m})$ SP that are observed as vestiges in the XRT imaging studies.

\section{CONCLUSION}

Vestiges of SP that were detected in these materials indicate that the SP prior to annealing were large and had a range in size (based on topographic imaging) of 100 to $500 \mu \mathrm{m}$. Sample MC3466 is a high performance material (based 
WSRC-STI-2008-0351

on its \%FWHM for an energy of $662 \mathrm{keV}$ ) but relative to the two other THM materials that we examined, it had a high amount of stress, crystalline strain, and considerably more SP that were visible with FTIR imaging (but not with XRT imaging). MC3466 also has defects such as a twin boundary or crack that is fairly evident in comparison to the features that were identified in the characterization of the other two THM grown Redlen samples. These various detectable features did not adversely influence radiation spectrometor performance in our study.

\section{ACKNOWLEDGMENTS}

This work was supported by the US DOE - National Nuclear Security Administration, through the Office of Nonproliferation Research and Development - NA-22 and National Science Foundation through the Fisk University Center for Physics and Chemistry of Materials (CPCoM), Cooperative Agreement CA: HRD-0420516 (CREST program) and through and from US DOE NA-22 Grant No. DE-FG52-05NA27035. Use of the Advanced Photon Source was supported by the U. S. Department of Energy, Office of Science, Office of Basic Energy Sciences, under Contract No. DE-AC02-06CH11357.

* Any mention of commercial products is for information only; it does not imply recommendation or endorsement by NIST.

\section{REFERENCES}

1. J.R. Heffelfinger, D.L. Medlin, and R.B. James. MRS Symp. Ser. 487, 33 (1998).

2. M. Schieber, T.E. Schlesinger, R.B. James, H. Hermon, H. Yoon, and M. Goorsky. J. Crystal Growth 237-239, 2082 (2002).

3. C. Szeles, and M.C. Driver. SPIE Proc. 3446, 1 (1998).

4. C. Szeles, S.E. Cameron, J-O. Ndap, and W.C. Chalmers. IEEE Trans. Nucl. Sci. 49, 2535 (2002).

5. J. Shen, D. K. Aidun, L. Regel, and W.R. Wilcox. J. Crystal Growth 132, 250 (1993).

6. G.A. Carini, A.E. Bolotnikov, G.S. Camarda, G.W. Wright, L. Li, and R.B. James. Appl. Phys. Lett. 88, 143515 (2006). 


\section{WSRC-STI-2008-0351}

7. A.E. Bolotnikov, G.S. Camarda, G.A. Carini, Y. Cui, L. Li, and R.B. James, Nucl. Instrum. Meth. A571, 687 (2007).

8. C. Szeles, W.C. Chalmers, S.C. Cameron, J-O. Ndap, M. Bliss, and K.G. Lynn. SPIE Proc. 4507, 57 (2001).

9. P. Rudolph, M. Neubert, and M. Mühlberg. J. Cystal Growth 128, 582 (1993).

10. P. Rudolph, A. Engel, I. Schentke, and A. Grochochi. J. Crystal Growth 147, 297 (1995).

11. R.S. Rai, S. Mahajan, S. McDevitt, and C.J. Johnson. J. Vac. Sci. Technol. B9, 1892 (1996).

12. L. Li, F. Lu, K. Shah, M. Squillante, L. Cirinano, W. Yao, R.W. Olson, P. Luke, Y. Nemirovsky, A. Burger, G.

Wright, and R.B. James. Nucl. Sci. Symp. Conf. Rec. IEEE 4, 2396 (2001).

13. H. Chen, S. A. Awadalla, J. Mackenzie, R. Redden, G. Bindley, A. E. Bolotnikov, G. S. Camarda, G. Carini, and R.

B. James. IEEE Trans. Nucl. Sci., 54, 811 (2007).

14. H. Chen, S.A. Awadalla, K. Iniewski, P.H. Lu, F. Harris, J. Mackenzie, T. Hasanen, W. Chen, R. Redden, G.

Bindley, I. Kuvvetli, C. Budtz-Jørgensen, P. Luke, M. Amman, J.S. Lee, A.E. Bolotnikov, G.S. Camarda, Y. Cui, A.

Hossain, and R.B. James. J. Appl. Phys. 103, 014903 (2008).

15. M. Ohmari, Y. Iwase, and R. Ohno. Mat. Sci. Eng., B16, 283 (1993).

16. R. Triboulet. Prog. Cryst. Growth Char. Matter, 128, 85 (1994).

17. D. Zeng, W. Jie, G. Zha, T. Wang, and G. Yang. J. Cryst. Growth, 305, 50 (2007).

18. M.C. Duff, D.B. Hunter, A. Burger, M. Groza, V. Buliga, J.P. Bradley, G. Graham, Z. Dai, N. Teslich, D.R. Black, and A. Lanzirotti, J. Materials Research (in review).

19. L.M. Bartlett, C.M. Stahle, D. Palmer, L.M. Barbier, S.D. Barthelmy, F. Birsa, N. Grehrels, J.F. Krizmanic, P. Kurczynski, J. Odom, A.M. Parsons, C. Sappington, P. Shu, B.J. Teegarden, and J. Trueller. Proc SPIE 2806,616 (1996).

20. H. Chen, K. Chattopadhyay, K.T. Chen, A. Burger, R.B. James, M.A. George, J.C. Gregory, J.J. Weimer, and P.K. Nag. J. Vac. Sci. Technol. A17, 1 (1997).

21. K.T. Chen, D.T. Shi, H. Chen, B. Granderson, M.A. George, W.E. Collins, A. Burger and R. James. J. Vac. Sci. Technol. A15, 1 (1997).

22. D.R. Rhiger and R.E. Kvaas. J. Vac. Sci. Technol. 21, 168 (1982).

23. T. Wang, W. Jie, and D. Zeng. Mat. Sci. Eng. 472, 227 (2008). 
WSRC-STI-2008-0351

a)

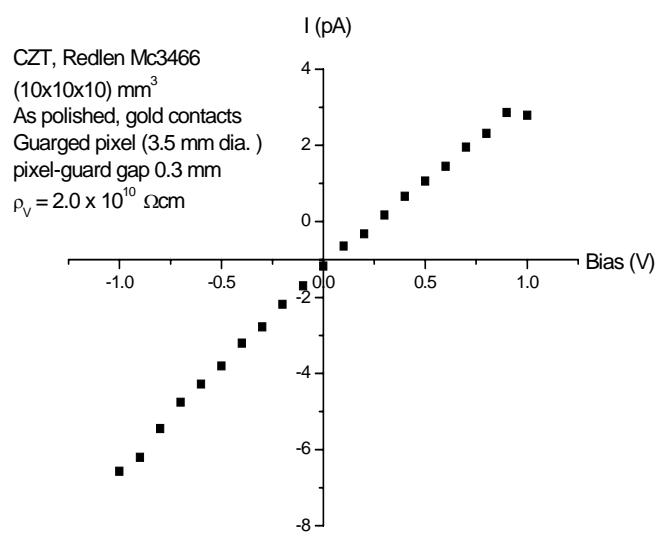

c)

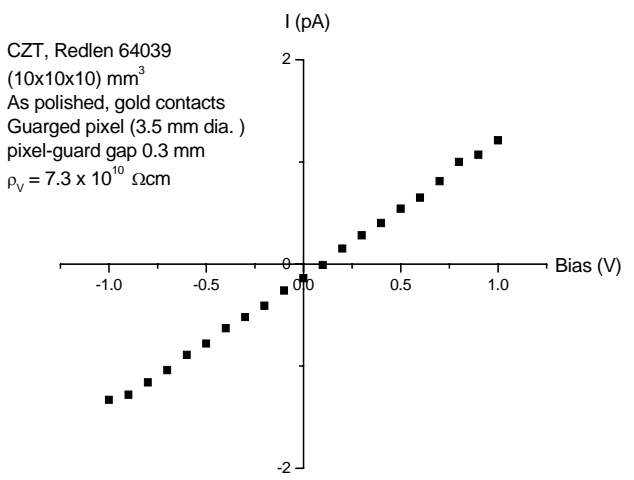

b)

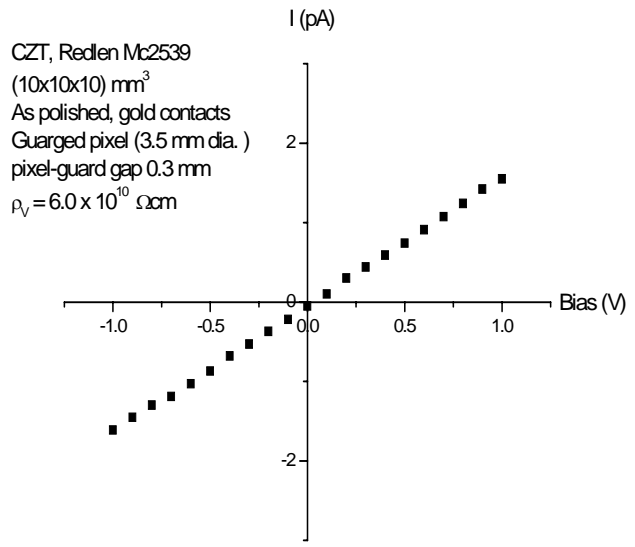

Fig. 1. Current-voltage characteristics for a) Redlen MC3466, $\rho_{\mathrm{V}}=2 \times 10^{10} \Omega \mathrm{cm}$; b) Redlen MC2539, $\rho_{\mathrm{V}}=6 \times 10^{10} \Omega \mathrm{cm}$; and c) Redlen $64039, \rho_{\mathrm{V}}=7.3 \times 10^{10} \Omega \mathrm{cm}$. 
WSRC-STI-2008-0351

a)

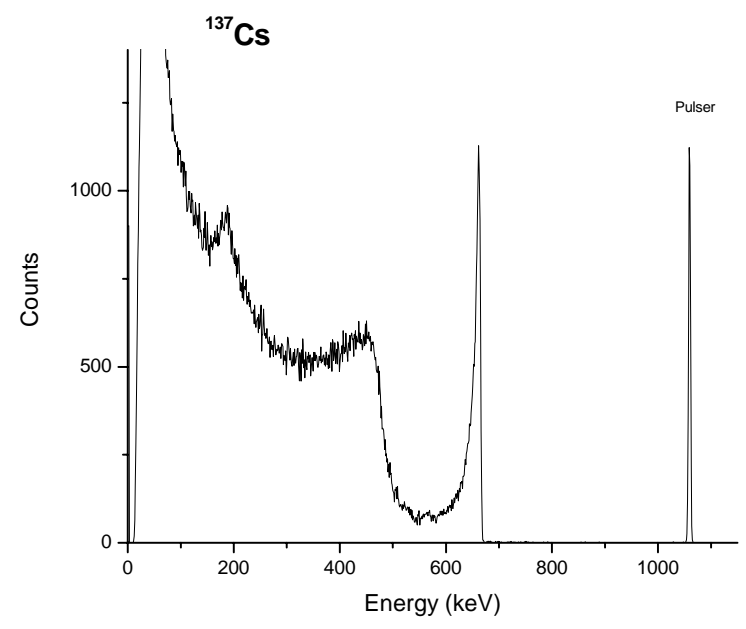

c)

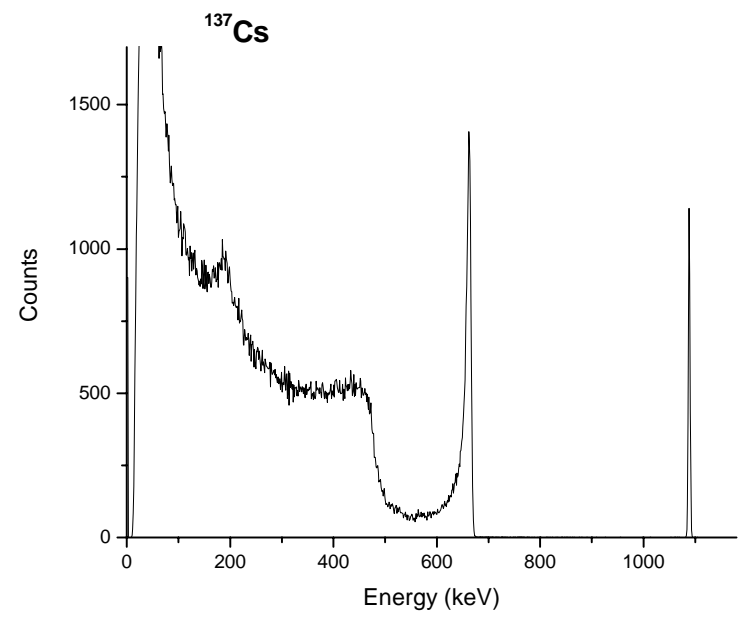

b)

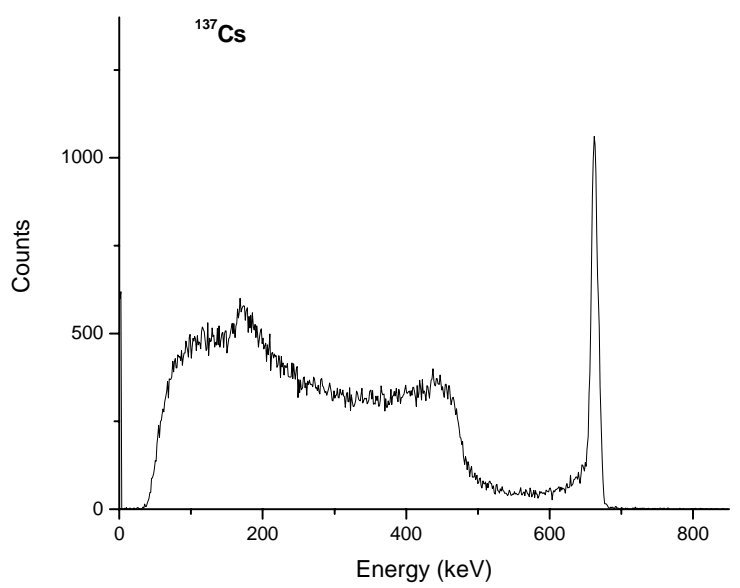

Fig. 2. Detector testing of Redlen CZT showing a ${ }^{137} \mathrm{Cs}$ energy spectrum for a) Redlen MC3466 with an energy resolution for $662 \mathrm{keV}$ is $1.5 \%$ and peak/Compton ratio 1.9; b) Redlen MC2539 with an energy resolution for $662 \mathrm{keV}$ is $1.8 \%$ and peak/Compton ratio 2.9; and c) Redlen 64039 with an energy resolution for $662 \mathrm{keV}$ is $1.6 \%$ and peak/Compton ratio 2.6 . 
a)

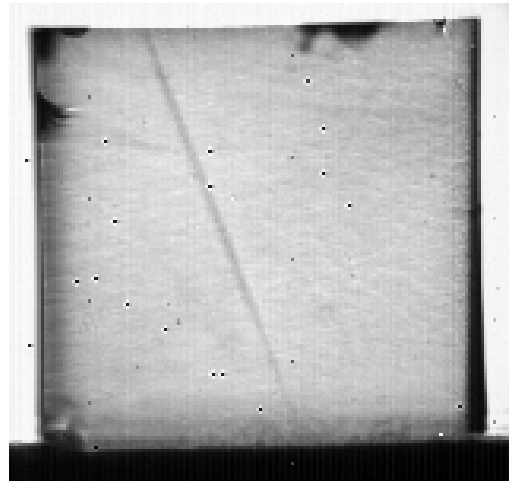

b)

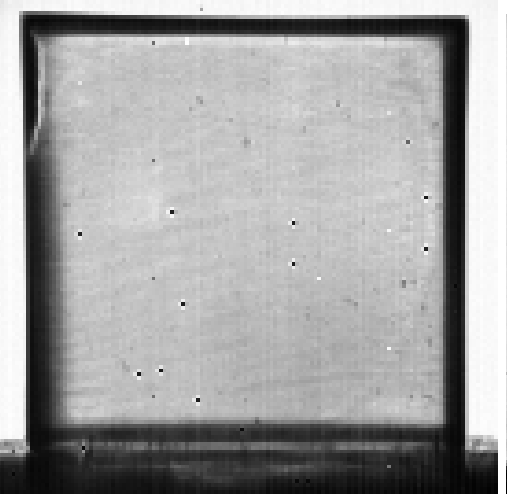

c)

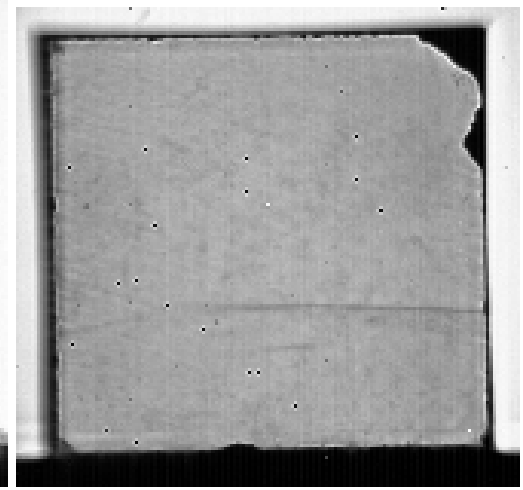

Fig. 3. IR transmission images (unpolarized light) of Redlen CZT: a) MC3466 b), MC2539 and c) 64039.

a)

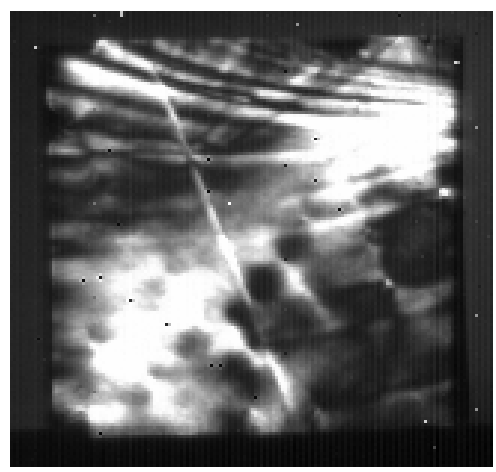

b)

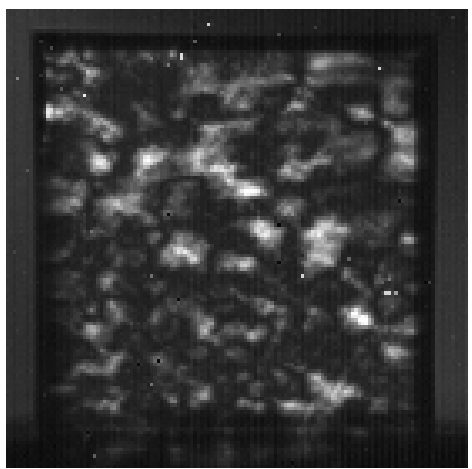

c)

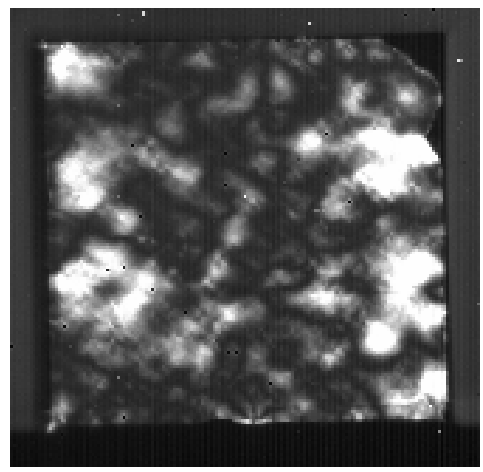

Fig. 4. Cross polarized IR transmission images $(\lambda=1150 \mathrm{~nm})$ of Redlen CZT, ID: a) MC3466, b) MC2539 and c) 64039. 
WSRC-STI-2008-0351

a)

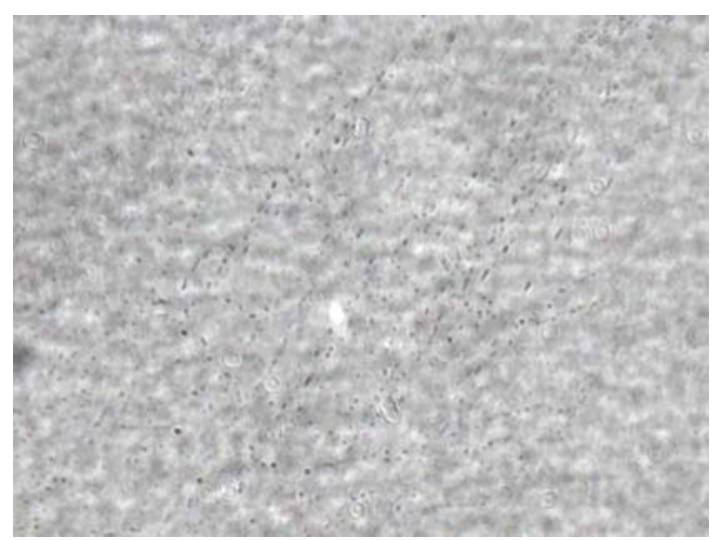

c)

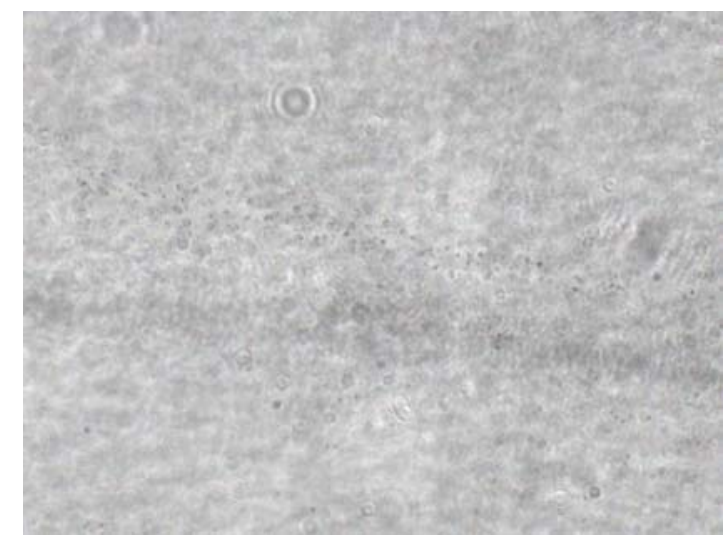

\section{$100 \mu \mathrm{m}$}

Fig. 5. IR microscopic imaging of internal detail ( $1.75 \times 1.3 \mathrm{~mm}^{2}$, depth of the field $\left.\sim 0.4 \mathrm{~mm}\right)$ of a) Redlen MC3466, b) Redlen MC2539 and c) Redlen 64039.

b)

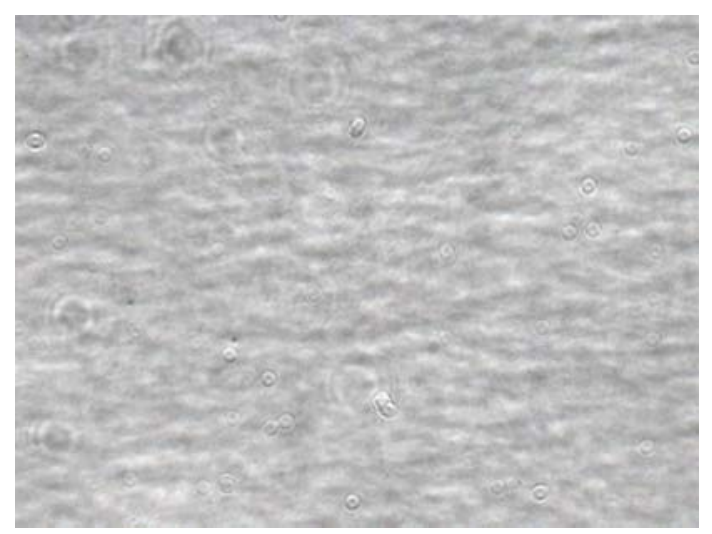
. 
a)

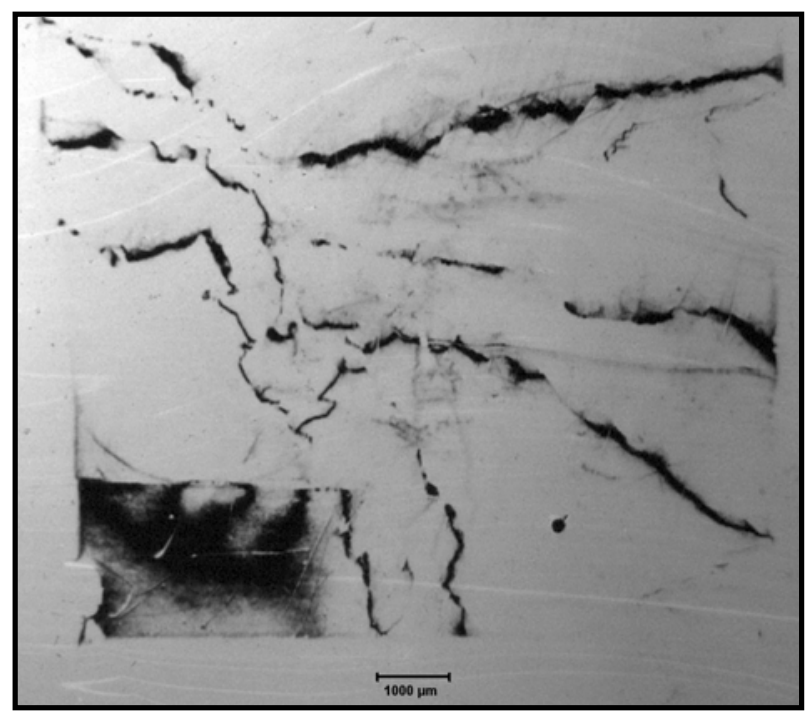

c)

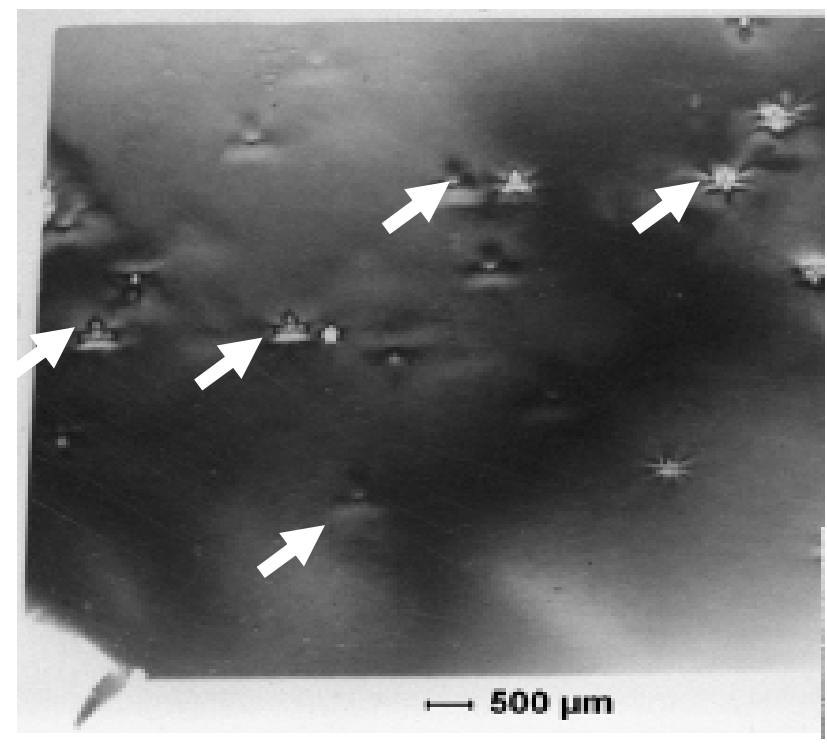

b)

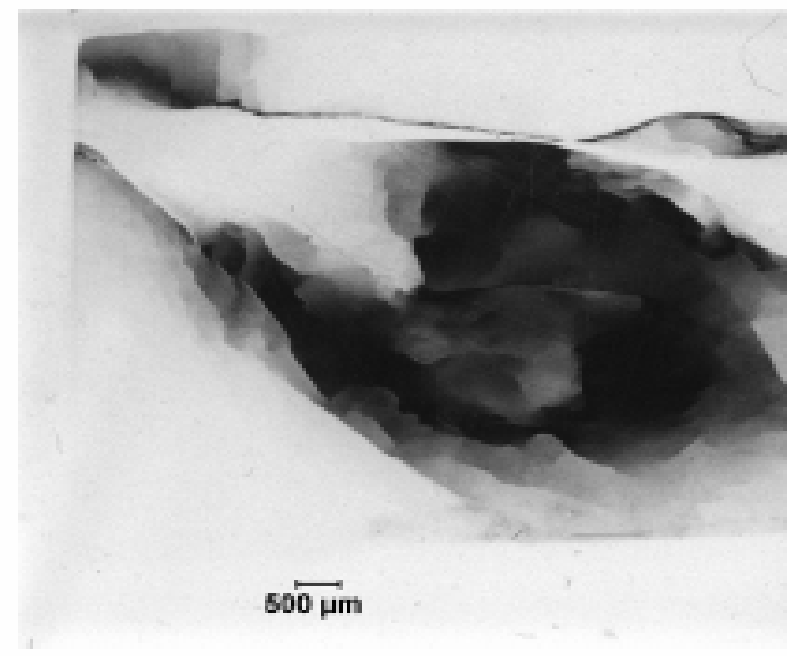

d)

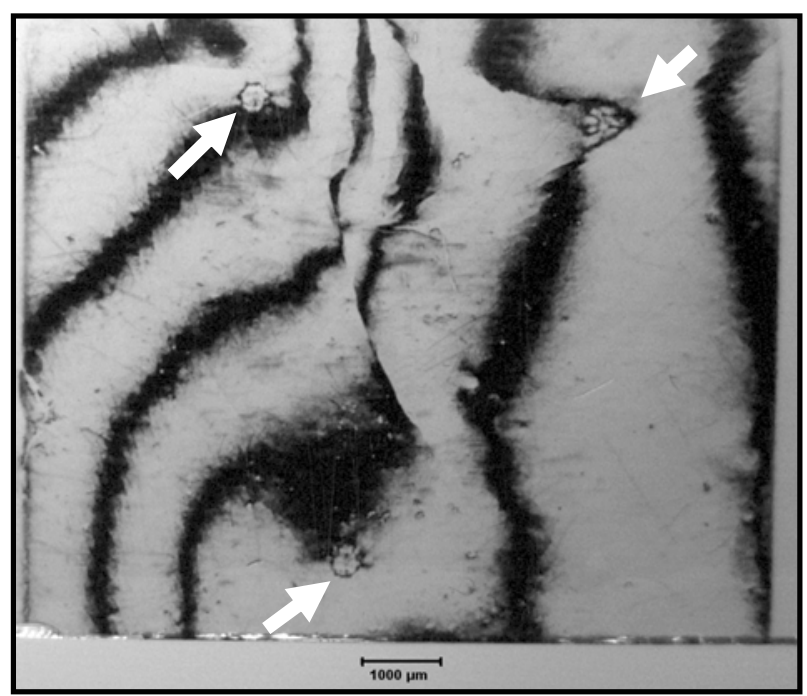

Fig. 6. X-ray topographs (all 333 symmetric images) of two different faces of MC3466 as shown in a) and b); c) sample 64039; and d) an X-ray topograph of MC2539. White arrows denote areas with vestiges of large triangular-shaped SP in c) and large hexagonal-shaped secondary phases in d). 
WSRC-STI-2008-0351

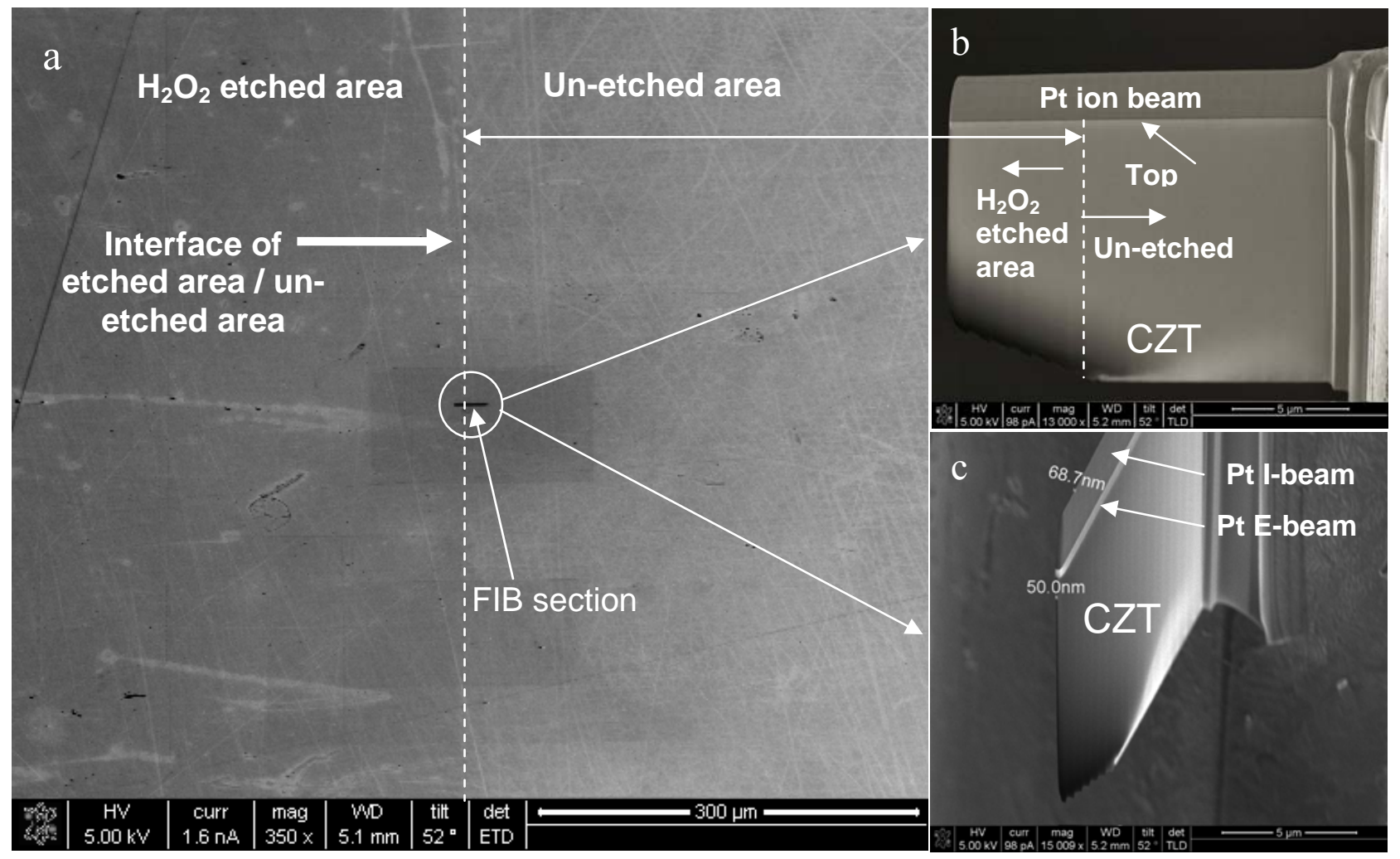

Fig. 7. SEM images of a) peroxide treated and untreated regions on MC2539 with images b) and c) showing two views of a thin section (removed by focused ion beam milling) from the region delineated in the circle as shown in a). 
a)
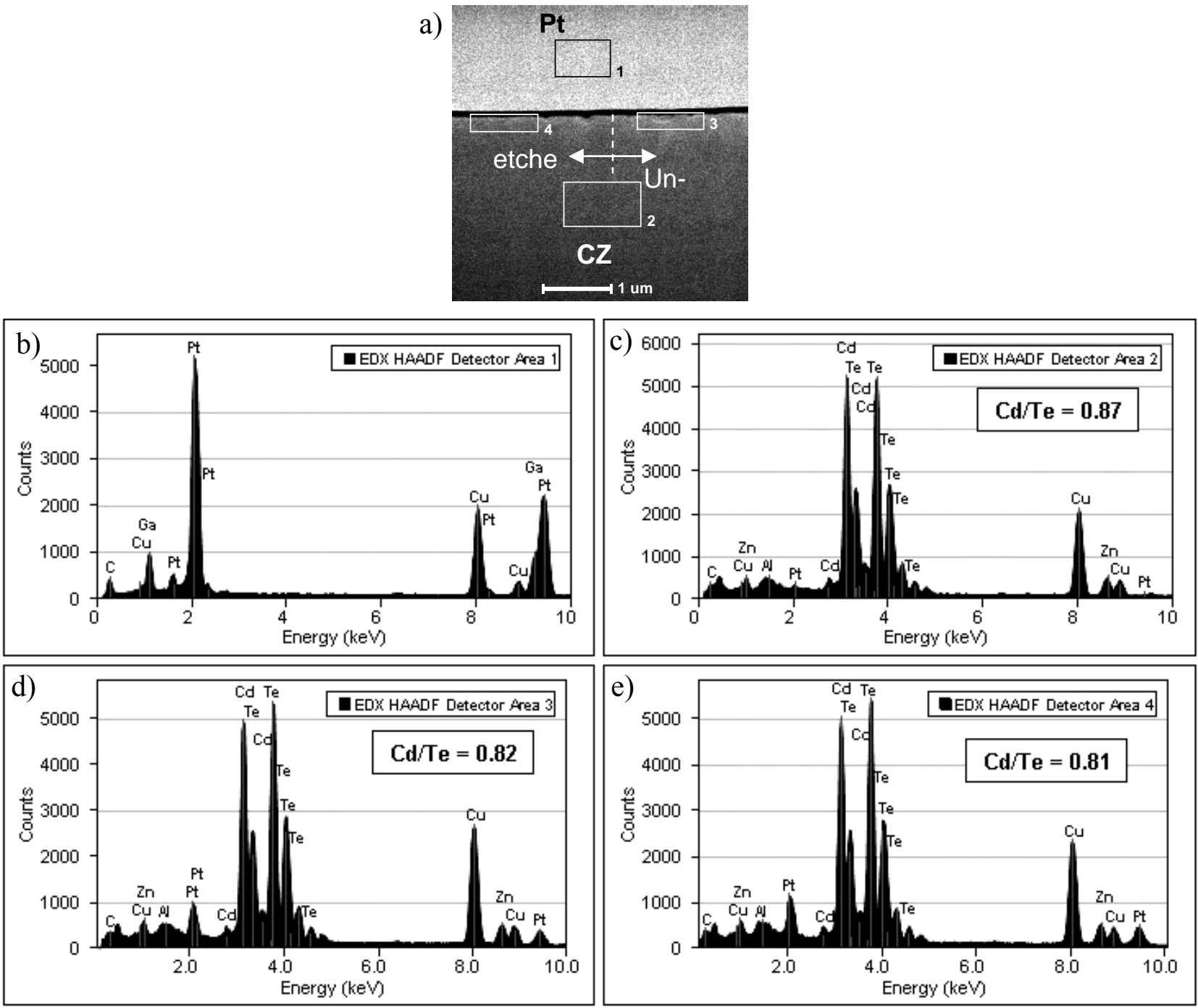

Fig. 8. STEM HAADF image of a) peroxide treated and untreated regions on MC2539 with energy dispersive spectra for b) the Pt coated sample; c) the bulk material, d) the unetched material and e) the peroxide etched material.

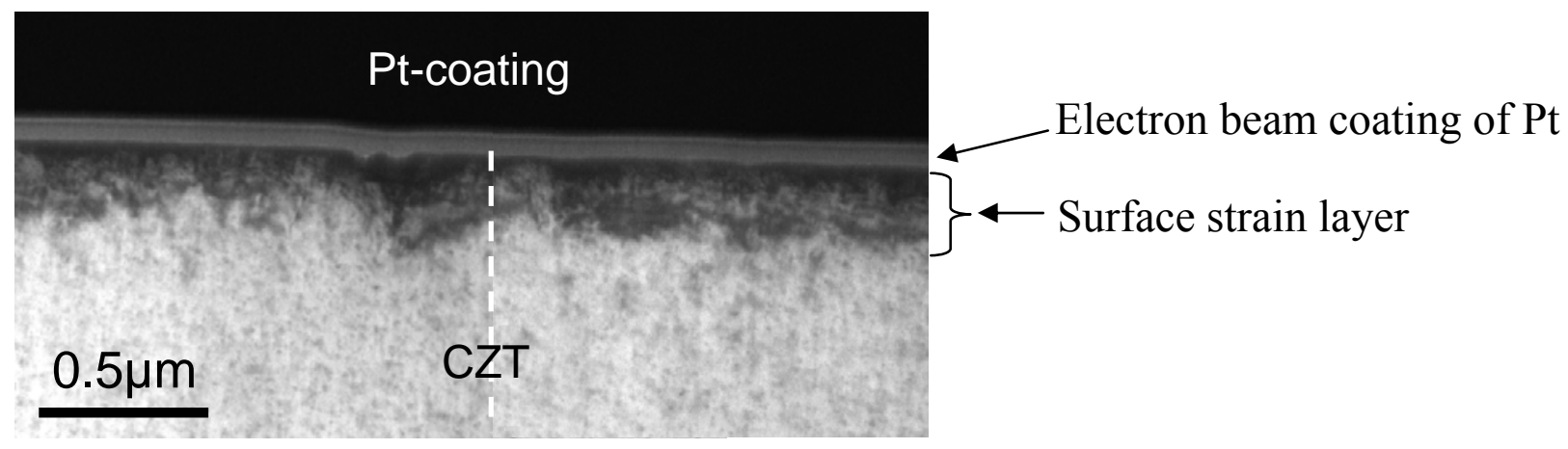

Fig. 9. Bright field TEM image of peroxide treated region (left half of image) and untreated (right half) region on MC2539. 